\title{
Reputation-based Joint Scheduling of Households Appliances and Storage in a Microgrid with a Shared Battery
}

\author{
Tarek AlSkaif ${ }^{\mathrm{a}, *}$, Adriana C. Luna ${ }^{\mathrm{b}}$, Manel Guerrero Zapata ${ }^{\mathrm{a}}$, Josep M. Guerrero ${ }^{\mathrm{b}}$, Boris Bellalta ${ }^{\mathrm{c}}$ \\ ${ }^{a}$ Department of Computer Architecture \\ Universitat Politecnica de Catalunya (UPC) \\ 08034 Barcelona, Spain \\ ${ }^{b}$ Department of Energy Technology \\ Aalborg University \\ 9220 Aalborg, Denmark \\ ${ }^{c}$ Universitat Pompeu Fabra (UPF) \\ 08018 Barcelona, Spain
}

\begin{abstract}
Due to the decreasing revenues from the surplus renewable energy injected into the grid, mechanisms promoting self-consumption of this energy are becoming increasingly important. Demand Response (DR) and local storage are among the widely used mechanisms for reaching higher self-consumption levels. Deploying a shared storage unit in a residential microgrid is an alternative scenario that allows households to store their surplus renewable energy for a later use. However, this creates some challenges in managing the battery and the available energy resource in a fair way. In this paper, a reputation-based centralized Energy Management System (EMS) is proposed to deal with these issues by considering households' reputations in the reallocation of available energy in the shared storage unit. This framework is used in an optimization problem, in which the EMS jointly schedules households' appliances power consumption and the energy that each household can receive from the storage unit. The scheduling problem is formulated as a Mixed Integer Linear Programming (MILP) with the objective of minimizing the amount and price of energy absorbed from the main grid. The MILP problem is coded in GAMS and solved using CPLEX. Numerical analysis is conducted using real data of renewable energy production and appliances' demand profiles for different classes of households and different annual periods in Spain. Simulation results of the different scenarios show that by using the proposed framework higher cost savings can be achieved, in comparison with the classical scheduling scenario. The saving can reach up to $68 \%$ when different classes of households exist in the microgrid. The results also show that the fairness in energy allocation is guaranteed by the reputation-based policy, and that the total power absorbed from the main grid by the whole microgrid is significantly decreased.
\end{abstract}

Keywords: Microgrids, self-consumption, energy management systems, demand response, photovoltaic, appliances scheduling, energy sharing, reputation-based systems.

\section{Introduction}

Microgrids are typically conceived as integrated operational and technological small-scale systems that help in optimizing power generation, distribution, and consumption. The concept refers to a set of loads (e.g., households), Distributed Generation (DG) (e.g., small-scale on-site Renewable Energy Sources (RESs)), and possibly Energy Storage Systems (ESSs) (e.g., batteries), operating as a single controllable system that provides power to its local area [1,2].

Since a large portion of electricity is consumed in the residential sector, involving citizens in the efficient planning and use of electricity is key. For instance, a $25 \%$ of the total electricity consumption in Spain is in the residential sector. More-

\footnotetext{
*Corresponding author. Tel.: +34603640 839

Email addresses: tarek@ac.upc .edu (Tarek AlSkaif), acl@et.aau.dk (Adriana C. Luna), guerrero@ac.upc.edu (Manel Guerrero Zapata), joz@et.aau.dk (Josep M. Guerrero), boris.bellalta@upf .edu (Boris Bellalta)
}

over, the share of electricity used by appliances and electronics in an average household accounts for around two-thirds of its total electricity consumption [3]. Hence, the management of households' appliances power consumption can play an important role in saving costs and reducing the environmental impact of the electricity consumed in the residential sector.

Accordingly, Demand Response (DR) programs have been defined, providing several economic and technical benefits for utilities and consumers [4]. Namely, DR programs aim to reshape consumer energy profiles in order to improve the reliability and efficiency of the grid and defer generation capacity expansion [5, 6]. Participants can take actions in response to a DR program by mean of load management schemes such as demand limiting, demand shedding, demand shifting and onsite generation [4]. Recently, an increasing focus of DR is placed on the residential sector motivated by the vision of future homes with smart appliances that allow their control and integration in Energy Management Systems (EMSs) [7]. DR can be performed as incentive-based or price-based programs [8]. 
Incentive-based schemes compensate participating users for demand reduction by offering discount rates separated to electricity prices [9]. Some examples of this kind of programs are Direct Load Control (DLC), interruptible/curtailable load, demand bidding and buyback, and emergency demand reduction [8]. Price-based schemes provide energy customers with time varying rates that define different electricity prices at different times. The customer reacts to the fluctuations in the electricity prices. This kind of programs might be confusing to customers, therefore scheduling techniques are needed to help customers manage their load [5]. Some of the implemented price-based schemes are Time of Use (ToU), Critical-Peak Price (CPP), and Real-Time Price (RTP) [8, 10]. RTP in DR programs are usually based on day-ahead or real-time wholesale price [11].

In [12], the potential benefits of DR on a residential distribution network operation are studied and the results show its influence in the load and voltage profiles, the network losses, and the service reliability. Still, some of the challenge of using DR in the residential sector are to establish an optimal DR system strategy beneficial for both customers and the utility, schedule demand in order to balance energy consumption with the available supply and implement the communication system that handles the DR [5]. To deal with these issues, some research has been conducted. In, [11], a dynamic DR controller is proposed to curtail peak load and save electricity under two RTP programs. The objective is to provide the set-point temperature for heating, ventilating and air conditioning (HVAC) systems based on the dynamic price of electricity and occupant preferences. In [13], two noncooperative games are defined to model a DR associated with the interaction among multiple utilities and customers in a smart grid. The first one, a supplierside game, defines the utility companies' profit maximization problem. The utility companies submit bids, then the electricity price is computed and sent to the customers. In the second game, a customer-side game, the price anticipating customers determine optimal shiftable load profile to maximize their daily payoff. In [14], the DR program is modelled as a repeated game with RTP scheme from the utility company perspective. The goal is to achieve a desired value for the peak to average ratio (PAR) in the aggregate load demand, and at the same time benefit the customers, by reducing their long-term cost. Nevertheless, those approaches are focused on the aggregated load and they do not considered the simultaneous management of other distributed energy resources.

The management of households' appliances and distributed energy resources has received significant attention in the last few years [15-|24]. In [15], the smart appliance power scheduling problem is modeled using Mixed Integer Linear Programming (MILP), capturing relevant appliance operational constraints. A distributed algorithm to schedule households' appliances aiming to minimize power costs by using game theory is presented in [16], where households are the players of the game and their strategies are the daily schedules of their appliances. In [17, 18], an ESS is used in the appliance scheduling problem, in which the battery charges from the main grid during off-peak times, and feeds the load during peak times. In [19], a residential energy consumption scheduling of electrical and thermal appliances to minimize energy costs of a customer with a RES is proposed taking its comfort into consideration. An artificial intelligence based smart appliance scheduling approach for reducing energy demand in peak periods by maximizing the use of RES in the residential sector is proposed in [20]. Other EMS that consider the ownership of both an onsite RES and an ESS in each household have been considered in [21-24]. However, equipping each household with an onsite ESS might be economically unaffordable due to the high cost of batteries which are required to buffer sufficient renewable energy for an average household daily power consumption [25]. Besides, batteries with long lifespan have a big physical size that makes them difficult to be located inside houses [26].

On the other hand, the increasing costs of electricity from the grid, the decreasing cost of photovoltaics (PV) technology and the expected decreasing revenues from excess electricity injected into the grid in the near future will raise the incentives to maximize the self-consumption ratio [27-29]. Moreover, in some cases, like the current situation in Spain, the surplus PV electricity injected into the grid is not remunerated and thus is lost for the household [29]. Therefore, new operation frameworks are needed in order to optimize the benefit from on-site RESs.

In this study, we consider a microgrid composed of households each with a PV system, that can inject the surplus PV energy into the main grid but without any compensation for it. To take advantage of this energy, a shared ESS is used (e.g., a battery), which is managed by a reputation-based EMS. The battery charges only from households surplus energy. In [30], a similar scenario is proposed with a more expensive electrical implementation and assuming the ESS as an inexhaustible energy resource that never gets fully charged or discharged. The reputation-based energy allocation policy is considered in the allocation of available energy in the shared battery, in a fair way, since they record the previous energy contribution of each household in charging the battery. This is more meaningful in a system where households' demands may exceed the available energy in the shared battery at some time periods. This framework is used in a daily appliances power scheduling optimization model, in which the EMS jointly schedules households appliances power consumption and the energy each household can receive from the shared battery, taking its operational constraints into account.

The contributions of this paper are summarized as follows:

- We propose a reputation function, according to which the EMS manages the available energy in the shared battery, and determines the portion of energy that will be scheduled to each household.

- We apply the proposed framework in a centralized optimization problem to minimize the energy absorbed from the grid in a DR scheme of RTP. The optimization model provides the power battery profiles as well as appliances power scheduling for each household.

The paper is structured as follows. The system model is presented in Section 2. The proposed reputation factor is described 


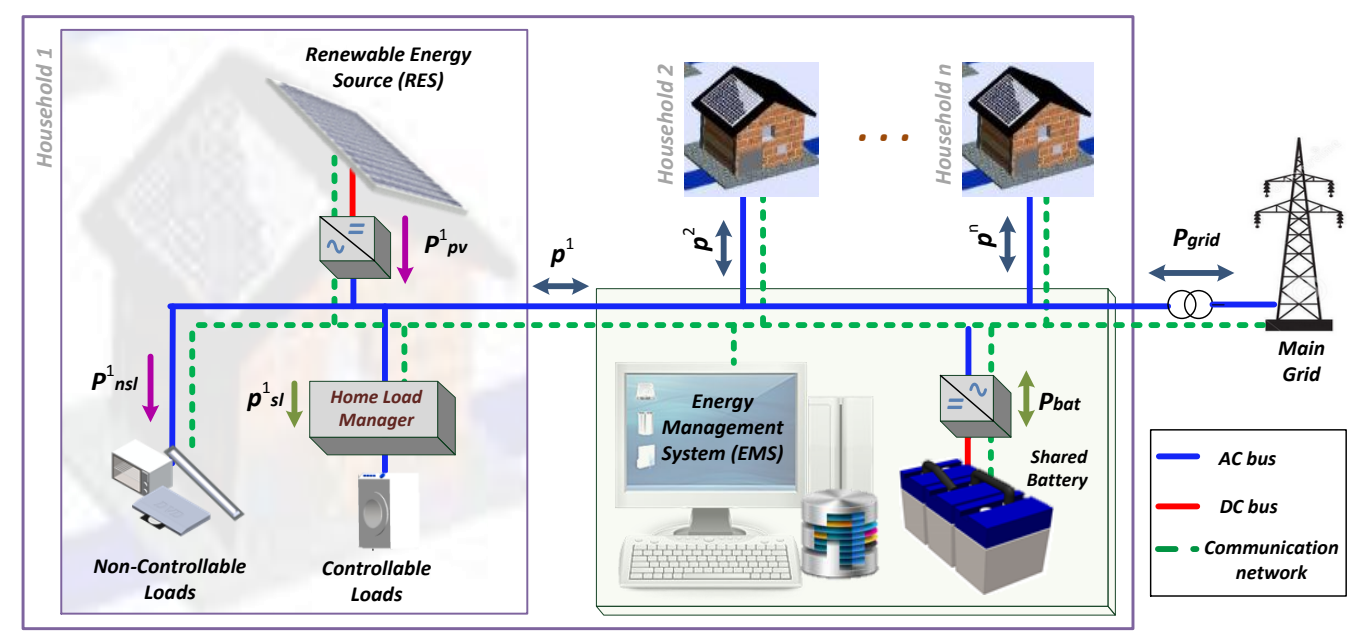

(a) Scheme of the considered microgrid.

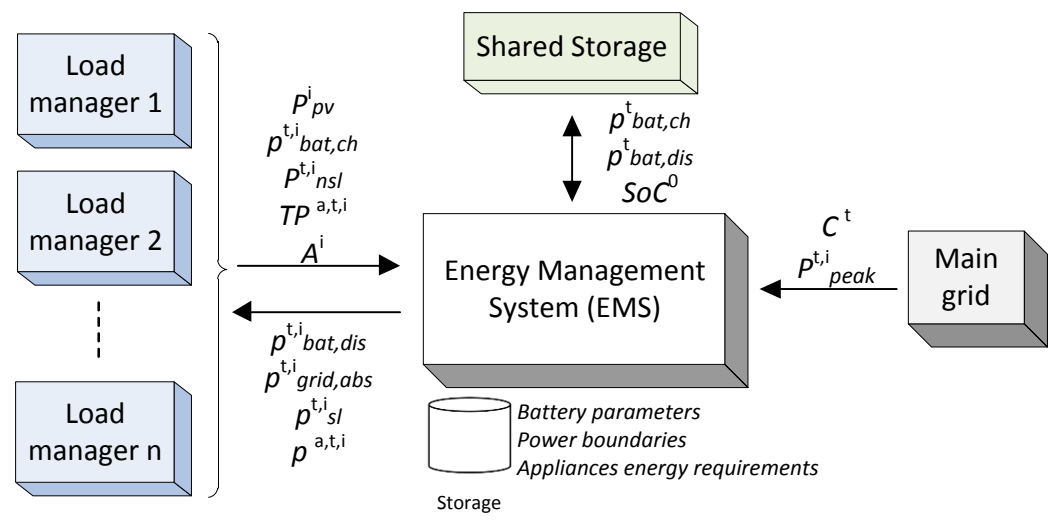

(b) Block diagram of the exchanged information with the centralized EMS.

Figure 1: System Architecture.

in Section 3 In Section 4, the household appliances power scheduling is presented and the centralized optimization problem is formulated in Section 5. Numerical results are discussed in Section 6. Finally, we conclude the paper and give pointers for possible future directions in Section 7

\section{System Model}

In this work we consider a generic microgrid which consists of a set of households $\mathcal{N}$, indexed by $i \in\{1,2, \ldots, N\}$, with a small-scale on-site RES (e.g., a solar PV system). Households are connected to the main grid and to the battery via AC power lines. They share their surplus harvested renewable energy by storing it in the shared battery that is controlled by an EMS. The EMS, in turn, controls the microgrid, manages households' demands, and allocates the shared renewable energy to them following an energy allocation policy. Households are connected to the main grid to secure their power demands during times of the day when renewable energy generation is impossible, when there is no available energy in the battery, or when the energy available in the battery is not scheduled.
We assume that households' demands are variable both in quantity and time. At a certain time period, each household could be a supplier which shares some amount of renewable energy, or a demander which requests some amount of energy from the battery. Each household is equipped with a load manager, which monitors and controls energy harvesting and power consumption intelligently. The load manager is also responsible for data communications between households and the EMS, as well as between households and the main grid.

The average power action of household $i$ happens on a time slot $t \in \mathcal{T}=\left\{t_{0}, t_{0}+\Delta t, t_{0}+2 \Delta t, \ldots, T\right\}$, and denoted as $p^{t, i}$. Each time slot can represent different timing horizons (e.g., an hour). In this way, the energy is represented by the average power during a time slot of length $\Delta t$ (i.e., $E=p \Delta t$ ). A power action of household $i$ at time slot $t$ could be either an interaction with main grid (i.e., injection $p_{\text {grid, inj }}^{t, i}$, or absorption $p_{\text {grid, abs }}^{t, i}$ ), or an interaction with the battery (i.e., charging $p_{\text {bat, ch }}^{t, i}$, or discharging $\left.p_{\text {bat, dis }}^{t, i}\right)$, where $p_{\text {grid, inj }}^{t, i}, p_{\text {grid, abs }}^{t, i}, p_{\text {bat, ch }}^{t, i}$ and $p_{\text {bat, dis }}^{t, i} \in \mathbb{R} . p_{\text {grid, inj }}^{t, i}$ is introduced to allow households to inject the excessive power into the main grid in case the battery is fully charged. The 
amount of power harvested by the local PV system of household $i$ at time slot $t$ is $P_{\mathrm{pv}}^{t, i}$. Besides $P_{\mathrm{pv}}^{t, i}$ and households' uncontrollable demand profile, the EMS receives additional information from the load manager of each household and from the utility. The load manager sends some input parameters about the number of appliances to be scheduled, $A^{i}$, and the time preference for the activation of those devices, $\mathrm{TP}^{a, t, i}$, where $a$ is the index of the corresponding controllable appliance. The utility sends the time varying electricity price at each $t, C^{t}$, and the maximum allowed power for the aggregated appliances demand in each time slot, $P_{\text {peak }}^{t, i}$. Other constant input data required to solve the optimization problem (e.g., power requirements of appliances) can be stored in the database of the EMS. By using all these data, the EMS calculates the set-points power of the battery and provides the optimal appliances schedules to load managers. The proposed system architecture and more details about the exchanged information with the EMS are illustrated in Fig. 1

\section{Reputation Factor}

In order to model the interaction between households and the EMS, and strengthen their cooperation, we define a reputation factor $R$ based on which the EMS will be able to dynamically and reliably allocate the available energy stored in the shared battery among households. Reputation-based systems belong to incentive-based mechanisms in cooperation enforcement games [31]. They have been proposed for similar engineering problems in P2P systems [32] and grid computing [33]. The basic idea is to identify entities based on their behavior. Entities that offer resources should be rewarded. On the other hand, selfish/unreliable entities should be gradually isolated from the system. In reputation-based systems, the actual value of an interaction depends heavily on the ability and reliability of involved entities. If each entity's history of previous interactions is made visible to the potential interaction partner, several benefits ensue. Firstly, a history may reveal information about an entity's ability, allowing to make choices about weather to interact with that entity, and on what terms. Secondly, an expectation that current performance will be visible in the future may deter the temptation to cheat or exert low effort in the present. Finally, because history reveal information about entities, entities with higher abilities will be drawn to participate, as they will be distinguishable from those of lower abilities, and respected or rewarded appropriately [34]. Reputation-based systems are a good application in energy sharing framework in microgrids, where there exists various classes of households with different power consumption profiles and when their aggregated demands may exceed the energy available in the shared battery.

In our proposed framework, the EMS keeps a reputation value for each household based on the amount of renewable energy it shared previously. As mentioned before, at each time slot $t$, household $i$ may charge or discharge the battery with an amount of power, $p_{\text {bat, ch }}^{t, i}$ or $p_{\text {bat, dis }}^{t, i}$, respectively. The reputation of $i$ depends on the total amount of renewable power it shared every day $d$ during a set of previous days $D^{p}$, being $p$ the last day of the set. It is denoted $R_{i}^{p}$ and calculated as follows:

$$
R_{i}^{p}=\frac{\sum_{d \in D^{p}} \sum_{t \in T} p_{\mathrm{bat}, \mathrm{ch}}^{t, i, d}}{\sum_{j \in N} \sum_{d \in D^{p}} \sum_{t \in T} p_{\mathrm{bat}, \mathrm{ch}}^{t, j, d} .}
$$

The value of the reputation factor $R_{i}^{p}$ represents the ratio between the total amount of renewable power shared by household $i$ during the set of previous days $D^{p}$, and the total renewable power shared by all households in the microgrid, including household $i$, during the same set $D^{p}$. In a similar way, the EMS calculates the reputation of other households. Reputations take positive values between 0 and 1 . The more renewable energy a household $i$ shares, the higher its reputation will be. This could motivate households to change their energy consumption behavior and/or share more renewable energy. A new household joins the system with a reputation equals to $1 / N$, which allows it to receive some amount of energy from the EMS.

\section{Household Appliances Power Scheduling}

Households' electric appliances are generally classified as cold appliances, cooking appliances, wet cleaning, electronics or miscellaneous [35]. They can also be divided into two categories: i) shiftable appliances, which can be run at flexible time schedule in scope of a day, or ii) non-shiftable appliances, which are uncontrollable and can not be scheduled. Wet cleaning electric appliances, including clothes washers, clothes dryers and dishwashers, are considered as shiftable appliances. Cold appliances (i.e., refrigeration) are typically considered as non-shiftable appliances in terms of, for example, their low capabilities for shifting power consumption for relatively long time periods. Nevertheless, those appliances have the potential to provide short-term flexibility through small adjustments of the on/off cycles while maintaining the temperature within limits [36]. The operation of non-shiftable and some shiftable appliances is typically uninterruptible, while some other shiftable appliances (e.g., pool pumps) can be interrupted.

Plug-in Electric Vehicles (PEVs) are emerging as more economic and environmentally-friendly alternatives to the conventional fossil fuel-based cars. When a large number of PEVs are integrated into the grid, the total charging demand constitutes a significant load which does not only increase the existing peak load demand, but may also introduce new peaks to the daily load profile [37]. DR and smart charging can play a major role in mitigating the effect of the increasing adoption of PEVs in households on the grid by providing proper incentives for shifting the charging times of PEVs. This control mechanism can reduce supply and customer side cost and enhance power system operating conditions [37]. Several studies have examined various aspects for PEVs charging scheduling in [21, 38, 41]. Game theoretic based scheduling approaches for addressing the overload problem associated with the charging demand of PEVs are proposed in [38, 39]. In [38], a stochastic model for the starting time of PEVs charging is given in order to simulate vehicle owners' charging behavior. A predictive approach for 
charging demand of the PEVs is provided in [41]. In [21, 40], the operation constraints of PEVs when working as distributed energy storage systems in a grid are described.

In this work we assume that each household $i$ has a number of shiftable appliances $A^{i}$ (i.e., including PEV) whose operation can be scheduled in the next 24 hours, where a time slot duration is one hour, in such a way that the cost of their total power consumption is minimized. The cost of $1 \mathrm{kWh}$ from the main grid at each time slot $t$ is assumed to be known (e.g., day-ahead RTP) and denoted as $C^{t}$. Some appliances might be used more than one time per day depending on the composition of the household and other factors. The operation happens in a time slot $t$ and may last more than one time slot per use according to appliances' characteristics. It is assumed that there is no sequential operation constraints between appliances and that each appliance has a predetermined daily energy requirement, a maximum and a minimum power per use (i.e., taken from appliances datasheet), and a maximum execution time. The maximum allowed power for the aggregated appliances demand in each time slot, $P_{\text {peak }}^{t, i}$, is also constrained.

As mentioned earlier, inside each household there is a load manager that controls the appliances and interacts automatically with the EMS to receive an optimal schedule for the shiftable load. The output of the EMS is the optimized power profiles of the scheduled appliances. Each appliance has a power profile denoted as $p^{a, t, i}$, corresponding to the power assigned to an appliance $a$ in household $i$ at time slot $t$. The power profile $p^{a, t, i}$ takes a real value and is measured in $\mathrm{kW}$ (i.e., it is written in small letters, since it will be considered as a decision variable in the appliances' optimization problem in Section 5. $p_{\mathrm{sl}}^{t, i}$ represents the total power demand of the scheduled shiftable appliances for household $i$ at time $t$. The total power of nonshiftable appliances (i.e., the basic load profile) at time $t$ for household $i$ is denoted $P_{\text {nsl }}^{t, i}$ (i.e., it is written in capital letters, since its value will be given as an input).

\section{Optimization Problem Formulation}

The following optimization problem is conceived as a MILP model that is performed by the EMS to jointly schedule households appliances power consumption and the energy that can be received from the shared battery in order to reduce appliances demand cost and minimize the power absorbed from the grid.

\subsection{Objective Function}

The objective function aims to minimize the amount of power absorbed from the main grid by each household, taking their reputations into account. It is defined as:

$$
\operatorname{minimize} \sum_{t=1}^{T} C^{t} \sum_{i=1}^{N} R_{i}^{p} p_{\text {grid, abs }}^{t, i} \Delta t,
$$

where $p_{\text {grid, abs }}^{t, i}$ is the power absorbed from the grid by household $i, C^{t}$ is the cost of power at time slot $t$, and $R_{i}^{p}$ is the reputation factor of household $i$. This factor is introduced to schedule the shared energy stored in the battery to each household proportional to its previous energy contribution in charging the battery. It is worth noting that minimizing the power absorbed from the grid implies taking benefit from the locally harvested solar energy, $P_{\mathrm{pv}}^{t, i}$ as well as from the scheduled energy provided by the shared battery, $p_{\text {bat, dis }}^{t, i}$. This will be illustrated in the following local balance constraints (i.e., Eq. 3 and 4 .

\subsection{Constraints}

\subsubsection{Local Balance}

The power balance between supply and demand should be assured in each household as follows:

$$
p^{t, i}=p_{\mathrm{sl}}^{t, i}+P_{\mathrm{nsl}}^{t, i}-P_{\mathrm{pv}}^{t, i}, \forall i, t,
$$

where $p^{t, i}$ is the average power action of household $i$ at time slot $t$ (i.e., as previously mentioned in Section 2 and seen in Fig. 11, namely:

$$
p^{t, i}=\left(p_{\text {grid, abs }}^{t, i}-p_{\text {grid, inj }}^{t, i}\right)+\left(p_{\text {bat, dis }}^{t, i}-p_{\text {bat, ch }}^{t, i}\right), \quad \forall i, t .
$$

\subsubsection{Global Balance}

The power exchange between households, the shared battery, and the main grid can be written as:

$$
\sum_{i=1}^{N} p^{t, i}=\left(p_{\text {grid, inj }}^{t}-p_{\text {grid, abs }}^{t}\right)+\left(p_{\text {bat, ch }}^{t}-p_{\text {bat, dis }}^{t}\right), \quad \forall t,
$$

where $\left(p_{\text {grid,inj }}^{t}-p_{\text {grid,abs }}^{t}\right)$ and $\left(p_{\text {bat, ch }}^{t}-p_{\text {bat, dis }}^{t}\right)$ represent the power interaction with the grid and the power available in the battery at time $t$, respectively.

\subsubsection{Grid Balance}

The households are exchanging power with the main grid and the battery at the same time. The contribution of each household in the whole system can be considered independently using the superposicion principle as shown in Fig. 2

In this way, the main grid global balance should be complied (Fig.2(a)), and it is formulated as follows,

$$
\sum_{i=1}^{N}\left(p_{\text {grid, inj }}^{t, i}-p_{\text {grid, abs }}^{t, i}\right)=\left(p_{\text {grid, inj }}^{t}-p_{\text {grid, abs }}^{t}\right), \quad \forall t .
$$

\subsubsection{Battery Balance}

Likewise, the battery global balance, illustrated in Fig.2(b), should be satisfied, and it can formulated as follows:

$$
\sum_{i=1}^{N}\left(p_{\text {bat, ch }}^{t, i}-p_{\text {bat, dis }}^{t, i}\right)=\left(p_{\text {bat, ch }}^{t}-p_{\text {bat dis }}^{t}\right), \quad \forall t .
$$

\subsubsection{Power Boundaries}

The variables related to the power absorbed from and injected to the main grid, as well as the power charges and discharges the battery, are bounded as follows:

$$
\begin{aligned}
& 0 \leq p_{\text {grid, abs }}^{t, i} \leq P_{\text {grid, abs }_{\max }}^{t, i}, \forall t, i, \\
& 0 \leq \quad p_{\text {grid, inj }} \leq P_{\text {grid, inj }_{\max }}^{i,}, \forall t, i, \\
& 0 \leq \quad p_{\text {bat, ch }}^{t, i} \leq P_{\text {bat, ch }_{\max }}^{i}, \forall t, i, \\
& 0 \leq \quad p_{\text {bat, dis }}^{t, i} \leq P_{\text {bat, dis }_{\max }}, \forall t, i,
\end{aligned}
$$




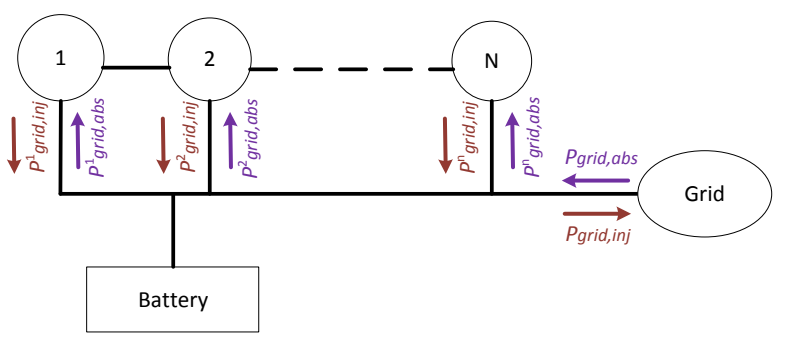

(a) Grid global balance (see Eq.6

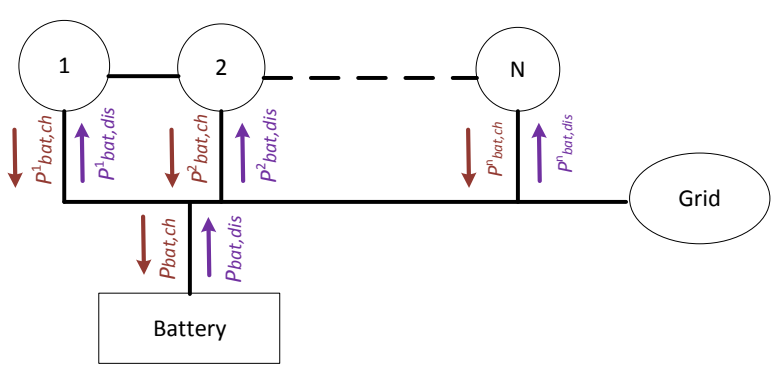

(b) Battery global balance (see Eq. 7 .

Figure 2: Power components flow in the main bus (see Eq. 5 .

where $P_{\text {grid, abs }}^{i}, P_{\text {grid, inj }}^{i}, P_{\text {max }}^{i}$ bat, dis max $_{\text {max }}$ and $P_{\text {bat, ch }}^{i}$ are constant values defined as boundaries for each household. They are related to the physical AC power lines capacity and battery operational constraints. In the case of the battery, those power limits are provided by the manufacturer for normal operation of the unit under both charging and discharging stage. The physical restrictions regarding the main grid are imposed by the system protector that exists inside each household such as fuse and circuit breakers. We note that the maximum amount of power that household $i$ can share with the battery, or inject into the grid should not exceed the total amount of solar energy it produces at time $t$ (i.e., $P_{\text {bat, ch }}^{i}=P_{\text {grid, inj }}^{i}=P_{\text {max }}^{t, i}$ ).

On the other hand, the power shared by each household with the battery should be safeguarded to ensure sharing only the energy produced by household's solar PV system (i.e., without charging the battery with any amount of power received from the main grid). This can be represented as follows:

$$
\begin{aligned}
P_{\mathrm{pv}}^{t, i}-\left(P_{\mathrm{nl}}^{t, i}+p_{\mathrm{sl}}^{t, i}\right) \leq \mathrm{M}\left(1-x^{t, i}\right), & \forall t, i, \\
p_{\text {bat, ch }}^{t, i}+p_{\text {grid, inj }}^{t, i}-P_{\mathrm{pv}}^{t, i}+\left(P_{\mathrm{nsl}}^{t, i}+p_{\mathrm{sl}}^{t, i}\right) \leq \mathrm{M} x^{t, i}, & \forall t, i, \\
p_{\text {bat, ch }}^{t, i}+p_{\text {grid, inj }}^{t, i} \leq \mathrm{M}\left(1-x^{t, i}\right), & \quad \forall t, i,
\end{aligned}
$$

where the binary variable $x_{i}^{t}$ is defined to determine when the generation is lower than the consumption for each household $i$. The value of M must be chosen sufficiently large so that the artificial variable would not be part of any feasible solution.

\subsubsection{Energy Storage System}

The battery's State of Charge ( $\mathrm{SoC})$ at time $t$ can be represented in terms of its power as the following:

$$
\begin{aligned}
\operatorname{SoC}^{t}=\operatorname{SoC}^{t-1}-( & \frac{1}{\eta_{\text {dis }} C_{\text {bat }}}\left(p_{\text {bat dis }}^{t}\right) \Delta t \\
& \left.-\frac{\eta_{\text {ch }}}{C_{\text {bat }}}\left(p_{\text {bat }, \mathrm{ch}}^{t}\right) \Delta t\right), \quad \forall t,
\end{aligned}
$$

where $\eta_{c h}$ and $\eta_{d i s}$ are the charge and discharge efficiency, respectively, and $C_{\text {bat }}$ is the battery's capacity that depends on the technology used.

The SoC of the shared battery is bounded as follows:

$$
\mathrm{SoC}_{\min } \leq \mathrm{SoC}^{t} \leq \mathrm{SoC}_{\max }, \forall t .
$$

Besides, a global balance of the battery should be included to ensure equal or better conditions for the next day:

$$
\sum_{t=1}^{T} \operatorname{SoC}^{t}-\operatorname{SoC}^{t-1} \geq 0, \forall t .
$$

\subsubsection{Shiftable Appliances Demand Management}

Part of the appliances demand is shiftable $\left(p_{\mathrm{sl}}\right)$ and can be scheduled to minimize costs.

\subsubsection{Daily power requirement}

This constraint ensures that the total energy assigned to each shiftable appliance per day fulfills its daily energy consumption requirement $E_{\mathrm{sl}}^{a}$.

$$
\sum_{t=1}^{T} p^{a, t, i} \Delta t=E_{\mathrm{sl}}^{a}, \quad \forall a, i .
$$

\subsubsection{Hourly demand}

This constraint indicates that the total power assigned to all shiftable appliances of household $i$ at a certain time slot $t$ is equal to its shiftable appliances demand at that time slot.

$$
\sum_{a=1}^{A^{i}} p^{a, t, i}=p_{\mathrm{sl}}^{t, i}, \quad \forall t, i
$$

\subsubsection{Power assignment bounds}

$$
P_{\min }^{a} y^{a, t, i} \leq p^{a, t, i}, \leq P_{\max }^{a} y^{a, t, i}, \quad \forall a, t, i,
$$

where $P_{\min }^{a}$ and $P_{\max }^{a}$ are the lower and upper limits of power assignment to an appliance $a$ which are taken from appliances datasheet, and $y^{a, t, i}$ is a decision binary variable that indicates whether an appliance $a$ at a particular time slot $t$ in household $i$ is switched on $\left(y^{a, t, i}=1\right)$ or off $\left(y^{a, t, i}=0\right)$.

\subsubsection{Peak power}

This constraint is to guarantee that the shiftable appliances demand of household $i$ in any time slot can not exceed an upper limit.

$$
p_{\mathrm{sl}}^{t, i} \leq P_{\text {peak }}^{t, i}, \forall a, i,
$$

where $P_{\text {peak }}^{t, i}$ denotes the peak signal determined by the utility ${ }_{392}$ company for each time slot $t$ and can also be considered as a ${ }_{393}$ DR signal. 


\subsubsection{Operation time}

Each household can set up a time preference constraint for each appliance. An appliance cannot be active outside its predetermined time preference interval.

$$
y^{a, t, i} \leq \mathrm{TP}^{a, t, i}, \quad \forall a, i,
$$

where $\mathrm{TP}^{a}$ represents the household's time preference for operating shiftable appliances in a certain day (e.g., the operation time of a PEV is between 19:00 and 07:00). Mathematically $\mathrm{TP}^{a}$ is a vector of 24 binary variables that are set by each household separately and take a value equal to one when it is preferable to household $i$ to switch an appliance $a$ at time slot $t$ and zero otherwise.

\subsubsection{Uninterruptible operation}

These constraints ensure a continuous operation of an appliance.

$$
\begin{aligned}
y^{a, t, i} \leq 1-z^{a, t, i} \quad \forall t, a, i, \\
y^{a, t-1, i}-y^{a, t, i} \leq z^{a, t, i} \forall t, a, i, \\
z^{a, t-1, i} \leq z^{a, t, i} \quad \forall t, a, i,
\end{aligned}
$$

where $y^{a, t, i}$ and $z^{a, t, i}$ are binary decision variables used to ensure that if an appliance $a$ starts working at a time slot $t$, it should not be interrupted until it finishes.

\section{Numerical Evaluation}

This section provides a performance evaluation of the proposed framework. First of all, we evaluate how the renewable energy is reallocated to each household based on its reputation. Then, we measure the economic impact of the proposed framework on the participating households. After that, we show how the system performance can be affected by the battery's capacity, the number of participating households, and the period of the year.

We consider a microgrid with $N=3$ households that share one battery. A time period represents one day and is divided to $T=24$ time slots. The performance of the proposed framework is measured by running the optimization model once at the beginning of the day (i.e., 24-hours ahead scheduling).

Since the power consumption in the residential sector can vary significantly among communities (i.e., tightly bounded with living habits and some social factors), we will run our simulations over households with different appliances demand profiles (i.e., different classes of households that are most common in Spain). The selected classes are listed in Table. 1.

\subsection{Renewable Power Profile}

It is assumed that the $N$ households have a solar PV system as an on-site RES, with the same capacity, material and installation settings, and that they generate a similar amount of renewable energy with a little variance (i.e., all houses are in the same area). Real hourly AC solar power measurements are used, which are outputted from a $1.5 \mathrm{~kW}$ solar PV system applied in Girona, Spain, during 2015 and with the characteristics
Table 1: The considered classes of households.

\begin{tabular}{llll} 
Class & $\begin{array}{l}\text { Household's } \\
\text { type }\end{array}$ & $\begin{array}{l}\text { Occupancy } \\
\text { pattern }\end{array}$ & Assumptions \\
\hline Class A & Two adults & $\begin{array}{l}\text { 18:00 to 9:00 } \\
\text { on weekdays }\end{array}$ & $\begin{array}{l}\text { Full-time working adults whose average } \\
\text { daily power consumption will be dis- } \\
\text { tributed throughout the day into two main } \\
\text { periods, from 6:00 till 9:00 and from } \\
18: 00 \text { till 01:00. }\end{array}$ \\
\hline Class B & $\begin{array}{l}\text { Two adults } \\
\text { with chil- } \\
\text { dren }\end{array}$ & $\begin{array}{l}\text { on weekdays } \\
\text { on }\end{array}$ & $\begin{array}{l}\text { One member has a full-time job and the } \\
\text { second adult holds a part-time job in the } \\
\text { morning in order to take care of the chil- } \\
\text { dren after school. }\end{array}$ \\
\hline Class C & $\begin{array}{l}\text { Two pen- } \\
\text { sioners }\end{array}$ & All the time & $\begin{array}{l}\text { Most loads are distributed throughout the } \\
\text { day in a random way and only what is re- } \\
\text { lated to cooking a specified periods. }\end{array}$ \\
\hline
\end{tabular}

Table 2: Solar PV system and performance data.

\begin{tabular}{|l|l|l|l|}
\hline Parameter & Value & Parameter & Value \\
\hline DC System Size (kW): & 1.5 & Location: & Girona, Spain \\
Module Type: & Standard & Array Type: & Fixed (roof mount) \\
Array Tilt (deg): & 20 & Array Azimuth (deg): & 180 \\
System Losses: & 14 & Invert Efficiency: & 96 \\
DC to AC Size Ratio: & 1.1 & & \\
\hline
\end{tabular}

listed in Table.2 Then, the renewable power of each household at each time slot is selected from a normal distribution with the mean value of the solar AC power output, and the standard deviation of $0.05 \mathrm{~kW}$.

\subsection{Appliances Demand Profile}

We develop an appliances demand profile generator similar to the one proposed in [42], which generates the average appliances power consumption profile for each class of households. The generator is based on a probabilistic model that predicts the possibility of each household to operate a certain amount of appliances on a certain time slot per day (e.g., there is a probability of 0.15 to run the dishwasher between 20:00-21:00, 0.3 between 21:00-22:00, 0.3 between 22:00-23:00, and 0.25 between 23:00-24:00 for households of class A). The appliances used in this tool, their power consumption, and their ownership level are compiled with respect to the statistical data provided by a study that analyses the energy consumption in the residential sector in Spain [3]. This generator provides quick and easy way to generate the average appliances demand profile of any class of households. It uses an hourly step calculator which we believe it is enough to provide a rough estimation of the daily appliances demand. We differentiate between household's appliances demand in weekdays and weekend. We also add some uncertainty in household's appliances demand during weekdays and weekends. A household's appliances demand at each time slot $t$ is selected from a normal distribution with the mean value of the appliances demand profile output, and a standard deviation of 0.1-0.15 kWh in weekdays and 0.3-0.4 in weekends.

Each of households' electric appliances in the model is assumed to have two operational states: on or off, and no appliance is left on standby. Cold appliances (i.e., refrigeratorsfreezers) are assumed to have a constant power demand when switched-on. Other appliances can be represented by timevarying demands. For example, a clothes washer that runs 


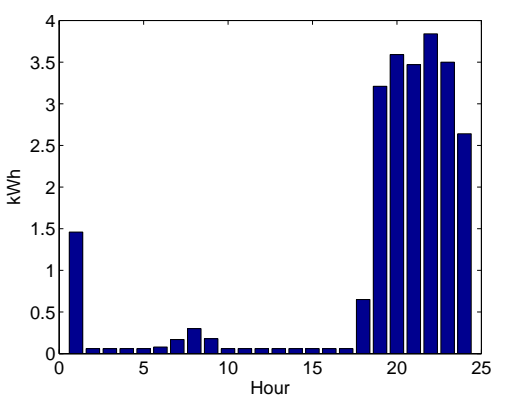

(a) Class A.

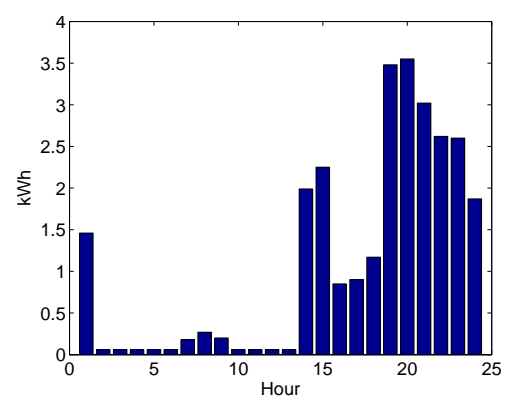

(b) Class B.

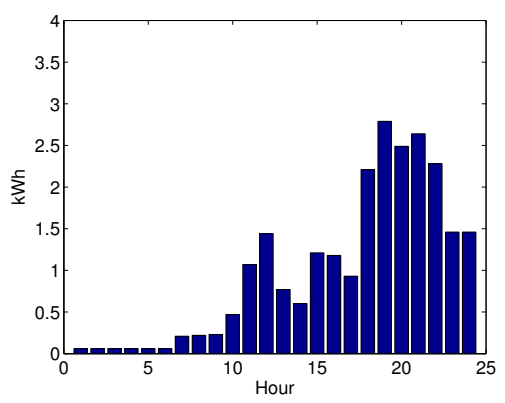

(c) Class C.

Figure 3: Average appliances demand profile of each considered class of households (without scheduling).

Table 3: The values of the problem parameters used in simulations.

\begin{tabular}{|c|c|c|c|}
\hline Parameter & Value & Parameter & Value \\
\hline$P_{\text {grid, abs }}^{i}(\mathrm{~kW})$ & 6 & $P_{\text {bat, dis }}^{i}(\mathrm{~kW})$ & 2 \\
\hline $\operatorname{SoC}_{\min }(\%)$ & 20 & $\mathrm{SoC}_{\max }(\%)$ & 100 \\
\hline $\mathrm{SoC}_{0}(\%)$ & 60 & $C_{\text {bat }}(\mathrm{kWh})$ & {$[5,30]$} \\
\hline$\eta_{\mathrm{dis}}$ & {$[0,1]$} & $\eta_{\mathrm{ch}}$ & {$[0,1]$} \\
\hline $\mathrm{TP}^{a, t, i}$ & $\begin{array}{l}\text { PEV: } 19: 00-07: 00 . \\
\text { DW, CW and CD: } \\
\text { 06:00-23:00 }\end{array}$ & $P_{\text {peak }}^{t, i}(\mathrm{KW})$ & 3.6 \\
\hline$P_{\min }^{a}=P_{\max }^{a}(\mathrm{~kW})$ & $\begin{array}{l}\text { PEV: } 1.4, \text { DW: } 0.625 \text {, } \\
\text { CW: } 0.67, \text { CD: } 1.39\end{array}$ & & \\
\hline
\end{tabular}

through various stages of water heating, washing and spinning, significantly varies its demand throughout a cycle. However, such detailed demand cycle data is not generally available. Thus, and for the sake of simplicity, we calculate appliances' demand per each time slot based on their total daily demand and operation period (e.g., if the washing machine is used one time per day, requires $1.34 \mathrm{kWh}$ per time of usage, and lasts two hours on average to complete its operation, then its demand per each time slot is $0.67 \mathrm{kWh}$ ). The weakly average appliances demand profiles of the selected classes of households are shown in Fig. 3 The selected household appliances and their daily average power consumption are presented in Table. 4 (i.e., for class $\mathrm{A}$ as an example). The listed operation times are the operation times in the ordinary case (i.e., without scheduling). It is assumed that all households in the considered microgrid have one of the listed appliances. In this numerical evaluation, each household has four shiftable smart appliances including a dishwasher (DW), a clothes washer (CW), a clothes dryer (CD), and a PEV.

\subsection{Simulation Results}

This section presents the simulation results of the optimization problem presented in Section V. The MILP problem is coded in GAMS 24.2.3 [43] and solved using IBM ILOG CPLEX Optimization Studio [44]. MATLAB R2014a is used as an interface. For the electricity pricing tariff, we use the RTP rate of the market in Spain in 2015 [45]. The execution period is from 00:00 till 24:00, and the length of time slots is 1 hour. The value of each parameter used in this simulation is provided in Table. 3 In this numerical evaluation, we consider
Table 4: Household appliances and their average energy consumption of Class A.

\begin{tabular}{|c|c|c|c|c|c|}
\hline \multirow{2}{*}{ Category } & \multirow{2}{*}{ Appliance } & \multirow{2}{*}{$\begin{array}{l}\text { Operation time } \\
\text { (most likely) }\end{array}$} & \multirow{2}{*}{$\begin{array}{l}\text { No. of times } \\
\text { per day }\end{array}$} & \multicolumn{2}{|c|}{ Average consumption } \\
\hline & & & & $\begin{array}{l}\text { per } \\
\text { capita } \\
(\mathrm{kWh} / \text { day }\end{array}$ & $\begin{array}{l}\text { per time } \\
\text { of usage } \\
(\mathrm{kWh})\end{array}$ \\
\hline \multirow{2}{*}{ Cooking } & Electric Oven & 18:00-22:00 & 1 & 1.00 & 2.00 \\
\hline & Microwave Oven & $\begin{array}{l}6: 00-9: 00 \text { and } \\
18: 00-22: 00\end{array}$ & 2 & 0.23 & 0.23 \\
\hline Refrigeration & Refrigerator-Freezer & All the day & 24 & 0.66 & 0.06 \\
\hline Electric Vehicle & PEV & 18:00-01:00 & 7 & 4.90 & 1.40 \\
\hline \multirow{3}{*}{ Wet Cleaning } & Clothes Washer $(\mathrm{CW})$ & $18: 00-24: 00$ & 1 & 0.67 & 1.34 \\
\hline & Clothes Dryer $(\mathrm{CD})$ & $19: 00-24: 00$ & 1 & 1.39 & 2.78 \\
\hline & Dishwasher (DW) & $20: 00-24: 00$ & 1 & 0.625 & 1.25 \\
\hline \multirow[t]{2}{*}{ Computers } & Desktop and Laptop & 19:00-24:00 & 5 & 0.40 & 0.16 \\
\hline & TV & 18:00-24:00 & 6 & 0.84 & 0.28 \\
\hline \multirow[t]{4}{*}{ Miscellaneous } & Electric Kettle & $\begin{array}{l}06: 00-09: 00 \\
\text { 19:00-20:00 }\end{array}$ & 3 & 0.39 & 0.26 \\
\hline & & $\begin{array}{l}\text { and } 22: 00- \\
24: 00\end{array}$ & & & \\
\hline & Iron & 18:00-24:00 & 1 & 0.09 & 0.18 \\
\hline & Others (e.g., Vacuum) & $18: 00-24: 00$ & 1 & 0.65 & 1.30 \\
\hline
\end{tabular}

that all households have the same protection system (i.e., the same power boundaries on the amount of power absorbed from the grid). Unless it is mentioned otherwise, we assume that the microgrid uses a battery of a $30 \mathrm{kWh}$ capacity with an initial SoC equal to $60 \%$, and an efficiency of charge and discharge equal to 1 .

In Fig. 4, the daily allocation of power by the shared battery $\left(\sum_{t=1}^{T} p_{\mathrm{bat}, \mathrm{dis}}^{t}\right)$ for each household during the first week of July 2015 is presented. The allocation of power for households of different classes, and of the same class (e.g., class A), are shown in Fig. 4 (a) and Fig. 4(b), respectively. The reputation is updated every day (i.e., $D^{p}=\{1\}$ ), and the total allocation is calculated at the end of the day. When households join the system, they start with an equal reputation. We set the initial reputation to $R=1 / N$. It is observed from Fig. 4 that the allocation of power strongly depends on households' reputations even if the differences in their reputations are small (see Fig. 4(b)). It is worth to highlight the correlation between the reputations, and the amount and distribution of appliances demand during the day (see Fig. 33). For instance, the appliances demand of households belonging to class $\mathrm{C}$ has a higher match with their solar PV energy generation profile than other classes of households. Therefore, their shared surplus renewable energy is less than other classes, which makes their reputation lower and their resulted allocation of power in future time periods smaller. The 


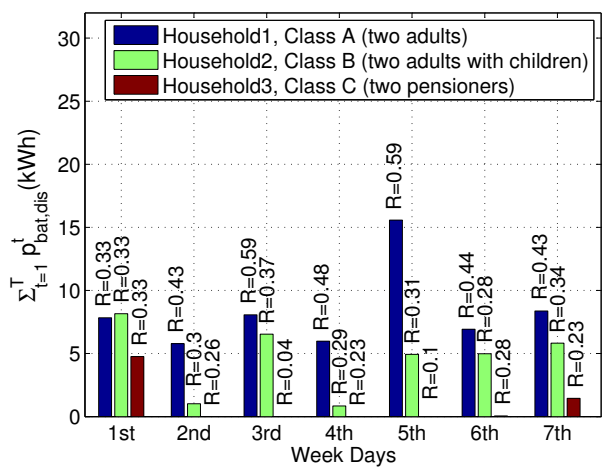

(a) Households of different classes.

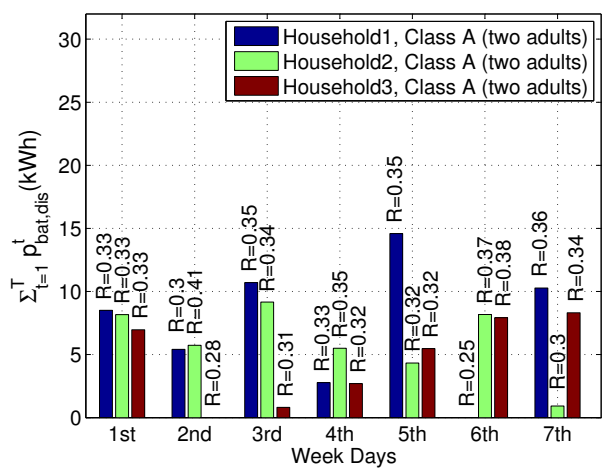

(b) All Households are of class A.

Figure 4: Daily amount of power received from the battery based on reputations during the first week of July 2015. (For interpretation of the references to color in this figure, the reader is referred to the web version of this article.)

MILP solver starts allocating the energy available in the battery to the household with the highest reputation, then it moves to next households in a descending order of reputation. When the battery does not have enough energy for all households, households with low reputation may not receive energy from the battery (e.g. household 3 of class C in Fig. 4(a)), although they share some amount of renewable energy every day. However, we argue that those households still have an interest to stay in the system, since they may share more energy in some time periods (e.g., if they go outside or if they are on vacations), and get a higher reputation in the next day.

It can also be noticed from Fig. 4 (4) that the amount of power allocated to the household of class A is always higher than to other classes, since their reputation is higher. This is because the amount of surplus renewable energy shared by households of class A is higher than other classes due to their occupancy pattern (i.e., from 18:00 to 9:00, see Table. 11). From Fig. 4tb), we notice that when all households are of the same class, their power allocation depends on the variability of their demands throughout the day and the uncertainty introduced from day to day.

In order to evaluate the economic impact of the proposed framework, we calculate and compare the appliances demand costs in three different scenarios. In the first scenario, the daily appliances demand costs are calculated in the ordinary case (i.e., without scheduling the shiftable appliances and without using the shared battery). In the second scenario, the shiftable appliances are scheduled at times when electricity tariffs are cheap, but without using the shared battery (i.e, which can be considered as a baseline to our framework). The third scenario captures the proposed framework, where both the shiftable appliances and the energy that each household can receive from the shared battery are scheduled. In all the scenarios, households satisfy their appliances demand from their solar PV system first.

In Fig. 5, the economic impact of the proposed framework on each participating household, represented by the average daily appliances demand cost and the average daily cost saving achieved, is presented. We run the three different scenarios in the first week of July 2015. Those scenarios are compared in two situations: i) when households are of different classes, in Fig. 5.a), and ii) when all households are of the same class: all of class A in Fig. 5. b), all of class B in Fig. 5. (c) and all of class $\mathrm{C}$ in Fig. 5(d). It is assumed that households are not enforced to make any additional payment for the power received from the shared battery.

We start by discussing the cost savings archived in the second scenario when the shared battery does not exist (i.e., green bars). In this scenario, Fig. 5(a) and (d) show that the cost saving achieved in class $\mathrm{C}$ households is higher than both class $\mathrm{A}$ and B, Fig. 5(b) and (c), respectively. This is related to the longer occupancy timeline of class $\mathrm{C}$ households than class A and B (see Table. 1). This gives class C households more flexibility for scheduling the shiftable appliances and results in a higher cost saving. We note that all the cost savings in this figure are with respect to the original cost (i.e., blue bars).

Fig. 5 shows that after applying the proposed appliances scheduling framework using the shared battery (i.e., the third scenario, red bars), the daily cost saving of appliances demand is noticeably increased. For instance, in Fig. 5 (a), when households are of different classes, up to $68 \%$ of saving is achieved by a class A household, which accounts for more than twice the saving achieved by the second scenario (i.e., green bar) in that situation. As illustrated before in Fig. 4 (4a), when all households are active, households with limited shared surplus energy (e.g., class C) achieves a limited cost saving due to their low reputation. However, we discussed earlier that those households still have some interest to stay in the system.

With respect to the second situation (i.e., when households are of the same class), the saving obtained by the proposed scenario when all households are of class A (i.e., Fig. 5(b)) is higher than when they are of class B and C, Fig. 5(c) and (d), respectively. The reason is that in those classes the occupancy timeline is longer than class $\mathrm{A}$, which results in higher matches between their appliances demand and their locally generated solar energy and lower amounts of surplus renewable energy shared with the battery. It is important to note that the differences between households' appliances daily cost and the achieved saving, when all belong to the same class, is due to the uncertainty of households' demand and generation profiles during the week (see Section 6.1 and 6.2.

Fig. 6 shows the system performance during different annual periods. In this simulation experiment, we consider a microgrid 


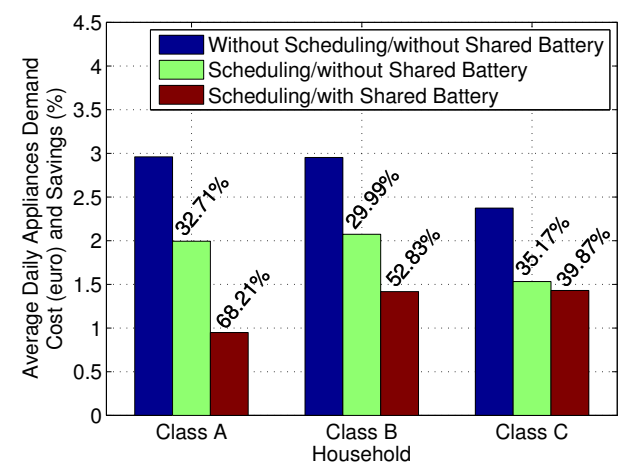

(a) Households are of different classes.

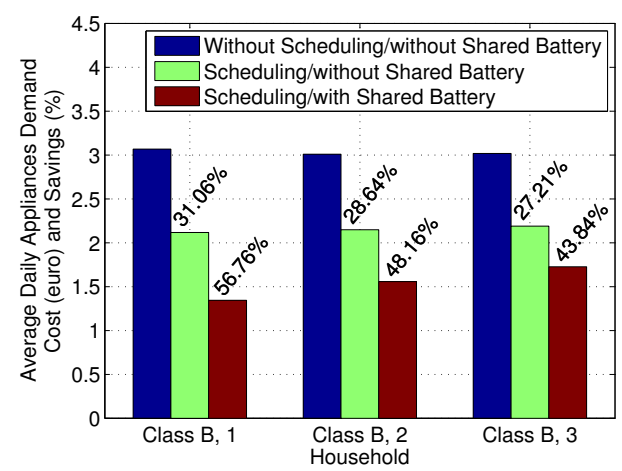

(c) All Households are of class B.

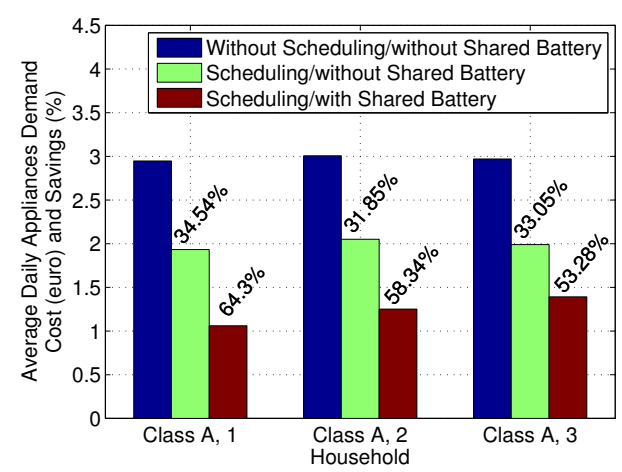

(b) All Households are of class A.

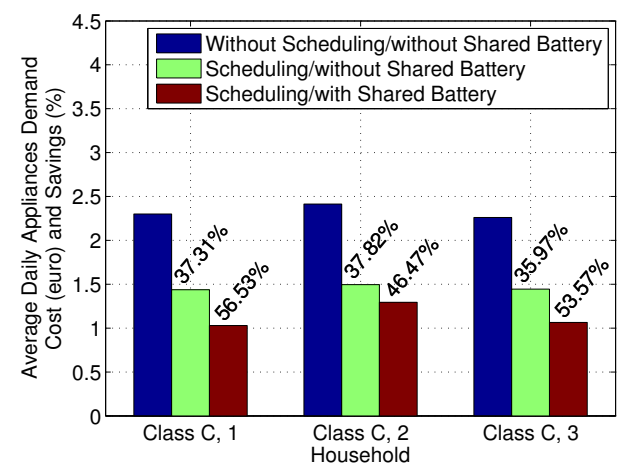

(d) All Households are of class C.

Figure 5: Households average daily appliances demand cost (euro) and the savings (\%) achieved in each scenario during the first week of July 2015. (For interpretation of the references to color in this figure, the reader is referred to the web version of this article.)

scenario that consists of three households of different classes. Fig. 6.a) illustrates how the average min and $\mathrm{max} \mathrm{SoC}$ reached (i.e., $S C_{\min }^{\text {reached }}$ and $\mathrm{SoC}_{\text {man }}^{\text {reached }}$, respectively) varies every month according to the amount of solar energy generated in Girona in 2015. Fig. 6(b) shows that the proposed framework reduces the total demands absorbed from the main grid by the whole $\operatorname{microgrid}\left(\sum_{t \in \mathcal{T}, i \in \mathcal{N}} p_{\text {grid, abs }}^{t, i}\right.$, see Eq. 6 .

From Fig. 6 a), we notice that in all the annual periods, the minimum $\mathrm{SoC}$ has not been reached (i.e., $\mathrm{SoC}_{\min }=20 \%$ in our simulation settings). This is because the EMS needs to guarantee a certain initial $\mathrm{SoC}$ at the beginning of next day (i.e., $\mathrm{SoC}_{0}=60 \%$ in our simulations, see Eq. 15 . In order to do that, the optimizer does not allow the battery SoC to go below a certain value, depending on the battery capacity, the amount of shared solar energy, and the number and class of participating households.

Therefore, we further study the effect of the battery capacity $C_{\text {bat }}$, and the number of participating households $N$ on $\mathrm{SoC}_{\min }^{\mathrm{reached}}, \mathrm{SoC}_{\text {man }}^{\text {reached }}$, and $\sum_{t \in \mathcal{T}, i \in \mathcal{N}} p_{\text {grid, abs }}^{t, i}$, by running the experiments presented in Table. 5. We assume that households are of different classes (i.e., Household 1,4 are of class A, 2,5 are of class B, and 3,6 are of class C) and all of them have the four shiftable appliances mentioned before. In this experiment, we have the same previous simulation settings except $\eta_{\mathrm{dis}}=0.9$ and $\eta_{\mathrm{ch}}=0.95$. Table. 5 shows how the system allows the battery to reach a lower $\mathrm{SoC}_{\min }^{\text {reached }}$ if its size is smaller or when the number
Table 5: The effect of the number of households and battery capacity on the min and max SoC and the total absorbed power from the main grid.

\begin{tabular}{|c|c|c|c|c|c|}
\hline \multirow{2}{*}{ Capacity $(\mathrm{kWh})$} & \multirow{2}{*}{ Parameter } & \multicolumn{4}{|c|}{ Number of households (N) } \\
\cline { 3 - 6 } & & 3 & 4 & 5 & 6 \\
\hline \multirow{3}{*}{$C_{\text {bat }}=30$} & $\sum p_{\text {grid, abs }}(\mathrm{kW})$ & 56.97 & 74.26 & 95.29 & 112.27 \\
\cline { 2 - 6 } & $\operatorname{SoC}_{\max }^{\text {reach }}(\%)$ & 83.66 & 94.02 & 95.57 & 94.83 \\
\cline { 2 - 6 } & $\operatorname{SoC}_{\min }^{\text {reached }}(\%)$ & 59.23 & 59.01 & 58.42 & 56.75 \\
\hline \multirow{3}{*}{$C_{\text {bat }}=15$} & $\sum p_{\text {grid, abs }}(\mathrm{kW})$ & 56.69 & 73.77 & 94.83 & 111.77 \\
\cline { 2 - 6 } & $\operatorname{SoC}_{\max }^{\text {reaced }}(\%)$ & 94.89 & 99.83 & 99.95 & 99.37 \\
\cline { 2 - 6 }$C_{\text {bat }}=7.5$ & $\operatorname{SoC}_{\min }^{\text {reached }}(\%)$ & 58.07 & 51.31 & 46.34 & 46.58 \\
\hline & $\sum p_{\text {grid, abs }}(\mathrm{kW})$ & 56.36 & 73.36 & 94.28 & 111.25 \\
\cline { 2 - 6 } & $\operatorname{SoC}_{\max }^{\text {reaced }}(\%)$ & 97.29 & 99.76 & 100.00 & 98.75 \\
\cline { 2 - 6 } & $\operatorname{SoC}_{\min }^{\text {reached }}(\%)$ & 54.29 & 39.36 & 41.56 & 38.30 \\
\hline
\end{tabular}

of households in the microgrid increases. The first case is due to the limited size of the battery. In this case, the system allows a lower $\mathrm{SoC}_{\min }^{\text {reached }}$, and at the same time it guarantees the required initial $\mathrm{SoC}_{0}$ at the beginning of the next day. The second case is because of the increased amount of shared renewable energy. It is clear in Table. 5 that the microgrid absorbs more power from the main grid as the number of households increases.

\subsection{Scalability and Computation Time}

In this section, the solving time of the scheduling optimization problem is computed for different number of households in the microgrid. The problem is run one time per day (i.e., 24-hours ahead scheduling). It is coded in GAMS 24.2.3 and 


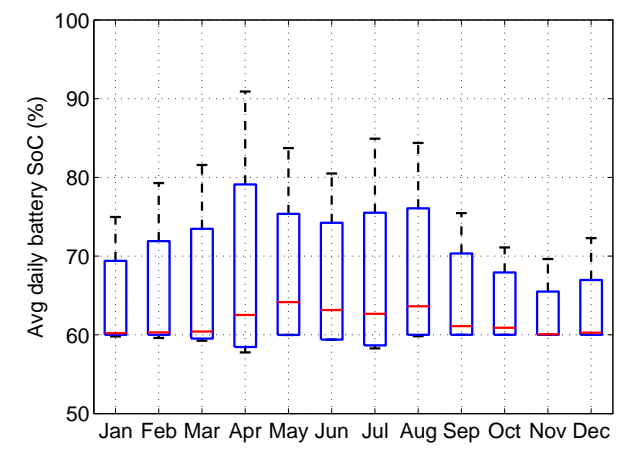

(a) Average min and max SoC reached.

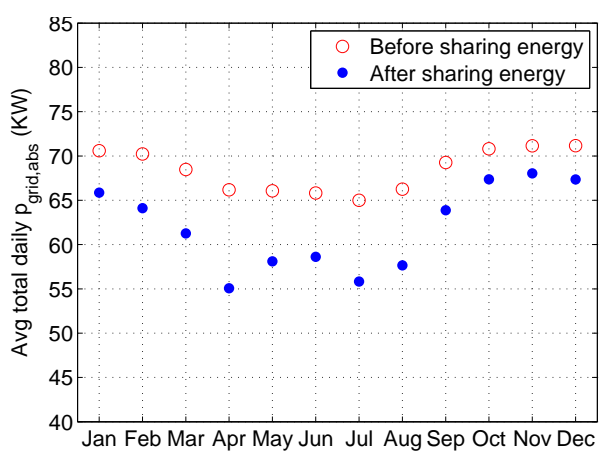

(b) Average amount of the total received power from the grid.

Figure 6: System performance during every month in 2015, (three households of class A, B and C).

solved using CPLEX 12 in a modern laptop (i.e., i7 at $2.4 \mathrm{GHz}$,

\section{GB of RAM, 64-bit Windows). We assume that the ownership} of shiftable appliances may differ from household to household, thus, the computation time for different number of shiftable appliances in each household is further calculated in each case. Fig. 7 shows how the computation time for solving the optimization problem changes in each case. It can be noticed that the number of households and the ownership of shiftable appliances have a significant impact on the computation time. However, the computation time remains reasonable when the number of households increases with a full ownership of the mentioned shiftable appliances.

\section{Conclusions}

In this study, a reputation-based centralized Energy Management System (EMS) for residential microgrids is proposed. Using this framework, households aim to maximize the selfconsumption of their on-site RES by storing their surplus renewable energy in a shared storage unit. The EMS runs a dayahead optimization problem to jointly schedule households' appliances power consumption and the energy that each household can receive from the shared battery. Households' reputations are considered by the EMS in the reallocation of available energy in the shared battery.

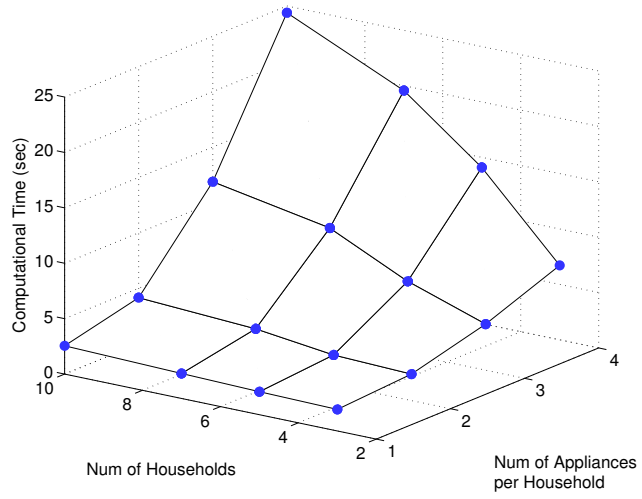

Figure 7: Computation time.

Simulation results assess the performance of this framework and show how households are able to achieve a cost saving of up to $68 \%$ by sharing only their surplus renewable energy. It is shown that their cost saving is tightly related with their reputation, that increases as they share more renewable energy. Using the reputation factors, the EMS will be able to fairly and reliably allocate the available energy stored in the shared battery among households. Further simulation experiments have been conducted to show the effect of the battery capacity and the number of participating households on the maximum and minimum battery's state of charge reached, and on the total amount of power absorbed from the main grid. In addition, we show that the problem solution can be obtained in a reasonable computation time for different number of households and different ownership level of shiftable appliances.

This study provides insights on how the shared energy using the reputation-based policy can be fairly and reliably allocated among households within the microgrid and how this framework can reduce power demands from the main grid without urging households to have a local ESS or to export electricity to the main grid. Future work will focus on applying this framework in real time which imposes additional supervisory control and prediction models. Selfish behavior and manipulation are also among the important issues that need to be considered in this reputation-based energy sharing framework.

\section{Acknowledgements}

This work was partially supported by projects TIN201347272-C2-2 and SGR-2014-881.

\section{References}

[1] H. Farhangi, The path of the smart grid, Power and Energy Magazine, IEEE 8 (1) (2010) 18-28.

[2] N. Hatziargyriou, H. Asano, R. Iravani, C. Marnay, Microgrids, Power and Energy Magazine, IEEE 5 (4) (2007) 78-94.

„1] 3$]$ P. Sech-Spahousec, Análisis del consumo energético del sector residencial en España (2011).

(1) URL http://www.idae.es/uploads/documentos/documentos_ informe_spahousec_acc_f68291a3.pdf 
[4] C.-J. Tang, M.-R. Dai, C.-C. Chuang, Y.-S. Chiu, W. Lin, A load control method for small data centers participating in demand response programs, Future Generation Computer Systems 32 (2014) 232-245.

[5] H. T. Haider, O. H. See, W. Elmenreich, A review of residential demand response of smart grid, Renewable and Sustainable Energy Reviews 59 (2016) 166-178.

[6] P. Siano, Demand response and smart grids: A survey, Renewable and Sustainable Energy Reviews 30 (2014) 461-478.

[7] B. Zhou, W. Li, K. W. Chan, Y. Cao, Y. Kuang, X. Liu, X. Wang, Smart home energy management systems: Concept, configurations, and scheduling strategies, Renewable and Sustainable Energy Reviews 61 (2016) 30-40.

[8] R. Deng, Z. Yang, M.-Y. Chow, J. Chen, A survey on demand response in smart grids: Mathematical models and approaches, IEEE Transactions on Industrial Informatics 11 (3) (2015) 570-582.

[9] J. S. Vardakas, N. Zorba, C. V. Verikoukis, A survey on demand response programs in smart grids: pricing methods and optimization algorithms, IEEE Communications Surveys \& Tutorials 17 (1) (2015) 152-178.

[10] Y. Liu, Demand response and energy efficiency in the capacity resource procurement: Case studies of forward capacity markets in iso new england, pjm and great britain, Energy Policy 100 (2017) 271-282.

[11] J. H. Yoon, R. Bladick, A. Novoselac, Demand response for residential buildings based on dynamic price of electricity, Energy and Buildings 80 (2014) 531-541.

[12] A. Safdarian, M. Fotuhi-Firuzabad, M. Lehtonen, Benefits of demand response on operation of distribution networks: a case study, IEEE systems journal 10 (1) (2016) 189-197.

[13] F. Kamyab, M. Amini, S. Sheykhha, M. Hasanpour, M. M. Jalali, Demand response program in smart grid using supply function bidding mechanism, IEEE Transactions on Smart Grid 7 (3) (2016) 1277-1284.

[14] S. Bahrami, V. W. Wong, An autonomous demand response program in smart grid with foresighted users, in: 2015 IEEE International Conference on Smart Grid Communications (SmartGridComm), IEEE, 2015, pp. $205-210$.

[15] K. C. Sou, J. Weimer, H. Sandberg, K. H. Johansson, Scheduling smart home appliances using mixed integer linear programming, in: Decision and Control and European Control Conference (CDC-ECC), 2011 50th IEEE Conference on, IEEE, 2011, pp. 5144-5149.

[16] A.-H. Mohsenian-Rad, V. W. Wong, J. Jatskevich, R. Schober, A. LeonGarcia, Autonomous demand-side management based on game-theoretic energy consumption scheduling for the future smart grid, Smart Grid, IEEE Transactions on 1 (3) (2010) 320-331.

[17] M. Rastegar, M. Fotuhi-Firuzabad, F. Aminifar, Load commitment in a smart home, Applied Energy 96 (2012) 45-54.

[18] D. Setlhaolo, X. Xia, Optimal scheduling of household appliances with a battery storage system and coordination, Energy and Buildings 94 (2015) 61-70.

[19] E. Shirazi, S. Jadid, Optimal residential appliance scheduling under dynamic pricing scheme via HEMDAS, Energy and Buildings 93 (2015) 40-49.

[20] B. Yuce, Y. Rezgui, M. Mourshed, ANN-GA smart appliance scheduling for optimised energy management in the domestic sector, Energy and Buildings 111 (2016) 311-325.

[21] N. G. Paterakis, O. Erdinc, A. G. Bakirtzis, J. P. Catalao, Optimal household appliances scheduling under day-ahead pricing and load-shaping demand response strategies, Industrial Informatics, IEEE Transactions on 11 (6) (2015) 1509-1519.

[22] M. H. Tushar, C. Assi, M. Maier, M. F. Uddin, Smart microgrids: Optimal joint scheduling for electric vehicles and home appliances, Smart Grid, IEEE Transactions on 5 (1) (2014) 239-250.

[23] J. Zhao, S. Kucuksari, E. Mazhari, Y.-J. Son, Integrated analysis of highpenetration PV and PHEV with energy storage and demand response, Applied Energy 112 (2013) 35-51.

[24] S. Shao, M. Pipattanasomporn, S. Rahman, Demand response as a load shaping tool in an intelligent grid with electric vehicles, Smart Grid, IEEE Transactions on 2 (4) (2011) 624-631.

[25] T. Zhu, A. Mishra, D. Irwin, N. Sharma, P. Shenoy, D. Towsley, The case for efficient renewable energy management in smart homes, in: Proceedings of the Third ACM Workshop on Embedded Sensing Systems for Energy-Efficiency in Buildings, ACM, 2011, pp. 67-72.

[26] X. Xie, Vanadium redox-flow battery, Tennessee Valley Authority.
[27] M. Castillo-Cagigal, E. Caamano-Martín, E. Matallanas, D. MasaBote, A. Gutiérrez, F. Monasterio-Huelin, J. Jiménez-Leube, PV selfconsumption optimization with storage and active DSM for the residential sector, Solar Energy 85 (9) (2011) 2338-2348.

[28] G. Merei, J. Moshövel, D. Magnor, D. U. Sauer, Optimization of selfconsumption and techno-economic analysis of PV-battery systems in commercial applications, Applied Energy 168 (2016) 171-178.

[29] G. Masson, J. I. Briano, M. J. Baez, Review and analysis of PV self-consumption policies, IEA Photovoltaic Power Systems Programme (PVPS) T1 (28)

[30] T. AlSkaif, M. G. Zapata, B. Bellalta, A reputation-based centralized energy allocation mechanism for microgrids, in: 2015 IEEE International Conference on Smart Grid Communications (SmartGridComm), IEEE, 2015, pp. 416-421.

[31] T. AlSkaif, M. G. Zapata, B. Bellalta, Game theory for energy efficiency in wireless sensor networks: Latest trends, Journal of Network and Computer Applications 54 (2015) 33-61.

[32] A. Satsiou, L. Tassiulas, Reputation-based resource allocation in P2P systems of rational users, Parallel and Distributed Systems, IEEE Transactions 21 (4) (2010) 466-479.

[33] N. Andrade, F. Brasileiro, W. Cirne, M. Mowbray, Discouraging free riding in a peer-to-peer cpu-sharing grid, in: High performance Distributed Computing, 2004. Proceedings. 13th IEEE International Symposium on Parallel and Distributed Processing with Applications, IEEE, 2004, pp. 129-137.

[34] N. Nisan, T. Roughgarden, E. Tardos, V. V. Vazirani, Algorithmic game theory, Vol. 1, Cambridge University Press Cambridge, 2007.

[35] R. Yao, K. Steemers, A method of formulating energy load profile for domestic buildings in the UK, Energy and Buildings 37 (6) (2005) 663671.

[36] I. Lampropoulos, N. Baghina, W. L. Kling, P. F. Ribeiro, A predictive control scheme for real-time demand response applications, Smart Grid, IEEE Transactions on 4 (4) (2013) 2049-2060.

[37] S. Gao, K. Chau, C. Chan, C. Liu, D. Wu, Optimal control framework and scheme for integrating plug-in hybrid electric vehicles into grid, Journal of Asian Electric Vehicles 9 (1) (2011) 1473-1481.

[38] S. Bahrami, M. Parniani, Game theoretic based charging strategy for plugin hybrid electric vehicles, IEEE Transactions on Smart Grid 5 (5) (2014) 2368-2375

[39] S. Bahrami, V. W. Wong, A potential game framework for charging PHEVs in smart grid, in: Communications, Computers and Signal Processing (PACRIM), 2015 IEEE Pacific Rim Conference on, IEEE, 2015, pp. 28-33.

[40] H. Fathabadi, Utilization of electric vehicles and renewable energy sources used as distributed generators for improving characteristics of electric power distribution systems, Energy 90 (2015) 1100-1110.

[41] M. H. Amini, A. Kargarian, O. Karabasoglu, ARIMA-based decoupled time series forecasting of electric vehicle charging demand for stochastic power system operation, Electric Power Systems Research 140 (2016) 378-390.

[42] F. Abd Jamil, A. Hashim, J. Cowie, D. Chukwu, I. Rizos, Demand Side Management: "Towards a Carbon Neutral Community" (2007).

URL http://www.esru.strath.ac.uk/EandE/Web_sites/0607/Carbon_neutral/START\%20PAGE.htm

[43] GAMS Development Corporation, Washington, DC, USA, General Algebraic Modeling System (GAMS). Release 24.2.3 (2013). URL http: //www.gams.com/help/index.jsp

[44] I. ILOG, CPLEX optimization studio (2014).

1 URL http://www-01.ibm.com/software/commerce/ optimization/cplex-optimizer

[45] Red Eléctrica de España, https://www.esios.ree.es/es/pvpc (2016). 